\title{
Litofácies, eletrofácies e petrofácies na caracterização de um reservatório carbonático Albiano do Campo A na Bacia de Campos
}

\author{
Mônica Tavares ${ }^{1 *}$, Nelson Franco Filho ${ }^{2} \&$ Abel Carrasquilla ${ }^{1},{ }^{1}$ UENF/CCT/LENEP, ${ }^{2}$ UOBC/Petrobras, Macaé - RJ, Brazil
}

Copyright 2018, SBGf - Sociedade Brasileira de Geofísica

Este texto foi preparado para a apresentação no VIII Simpósio Brasileiro de Geofísica, Salinópolis, 18 a 20 de setembro de 2018 . Seu conteúdo foi revisado pelo Comitê Técnico do VIII SimBGf, mas não necessariamente representa a opinião da SBGf ou Técnico do VIII SimBGf, mas não necessariamente representa a opinião da SBGf ou
de seus associados. É proibida a reprodução total ou parcial deste material para propósitos comerciais sem prévia autorização da SBGf.

\begin{abstract}
In this study, the lithofacies, electrofacies and petrofacies criteria were used to characterize an Albian carbonate reservoir of the Oilfield $A$ in Campos Basin. Several laboratory measurements and basic geophysical logs of two-wells were analyzed. By interpreting all this information, it is observed that the lithofacies essentially reflect the depositional control of the rock without including in its textural classification the diagenetic phenomena. It can then be considered that, for zones with samples not affected by diagenesis, there is a good correlation between the grainstone with good petrofacies, packstones with regular petrofacies and mudstones/wackstone with poor petrofacies.
\end{abstract}

\section{Introdução}

As rochas carbonáticas possuem aspectos únicos e distintos das outras rochas. São rochas sedimentares, de origem clástico, química ou bioquímica, composta principalmente por calcita. Avaliar a origem, composição e variação pós deposicional é muito importante para analisar a estrutura dessas rochas e, assim, desenvolver modelos de reservatório que descrevam de forma confiável este sistema. Considerando que estas rochas representam grande parte das reservas mundiais de óleo e gás, ser capaz de definir o tipo de fluidos e a quantidade de hidrocarbonetos contidos nos reservatórios é de fundamental importância para o planejamento da comercialidade ou abandono de um poço, por exemplo (Lucia, 2007).

Um termo muito utilizado na caracterização de reservatórios é o da litofácies, que é um tipo de rocha caracterizada dentro de um ambiente geológico pela sua composição mineralógica, arranjo textural, modelo deposicional (processos físicos, energia do ambiente de deposição e a acomodação de grãos em um substrato). Entretanto, não incluem na sua classificação textural os fenômenos diagenéticos que ocorrem nos grãos e entre os grãos, que são posteriores à deposição (processos químicos e mecânicos) (Serra e Abbott,1982).

Quando é possível a extrapolação das fácies individualizadas a todas as partes do reservatório utilizando perfis geofísicos de poços, assumem-se nomes como eletrofácies ou petrofácies, que podem representar as fácies em seus ciclos deposicionais e, também, suas propriedades permoporosas avaliadas pelos perfis (Ellis \& Singer, 2007).

Eletrofácies é o conjunto de respostas de perfis que caracteriza um sedimento e permite sua distinção dos demais (Serra, 1986). Devido à importância e aplicabilidade de estudos que contemplam a caracterização de eletrofácies e dados geológicos diretos, cada vez mais este tema vem sendo estudado dentro da modelagem geológica de reservatórios (Schlumberger, 1987).

As petrofácies possuem a mesma definição que a eletrofácies, porém trata-se de uma descrição acrescida da caracterização petrofísica de laboratório como ensaios de porosidade e permeabilidade a gás, pressão capilar e outros. As petrofácies devem caracterizar a geometria porosa, a saturação de fluidos e sua distribuição em um determinado reservatório, sendo classificada a partir de diversas medições petrofísicas. Ela pode identificar a estrutura porosa, conectividade e suas particularidades com relação ao fluxo de fluidos. Também, relaciona as características permoporosas que resultam do controle deposicional (colapso mecânico do arcabouço, dissolução de grãos e/ou matriz e precipitação de material secundário), procurando identificar melhores e piores condições de armazenamento e fluxo de fluidos oriundos das rochas (Doveton,1994).

O termo petrofísica refere-se as propriedades físicas inerentes a todos os tipos de rochas e seus constituintes como, por exemplo, porosidade, permeabilidade, densidade, dureza, velocidade do som, etc. A classificação de uma rocha reservatório, ou simplesmente a tipificação da rocha, tem sido reconhecida como uma das ferramentas mais eficazes para facilitar a modelagem e simulação de reservatórios (Rider, 2000).

\section{Metodologia}

O conjunto de dados utilizados compreendem: 13 ensaios laboratoriais de pressão capilar por injeção de mercúrio; 493 medidas de petrofísica básica de porosidade e permeabilidade (154 pertencentes ao poço X3 e 339 ao poço X10); perfis geofísicos básicos (raios gama - GR, sônico - DT, resistividade - ILD, densidade - RHOB e porosidade neutrônica - NPHI); curva de eletrofácies (EFAC), definida da análise discriminante com a supervisão das litofacies descritas nos testemunhos; curva de porosidade (PHIT) derivada do perfil RHOB; e, curva de permeabilidade sintética (Ksint), gerada por uma regressão linear múltipla para de ambos poços separadamente e em conjunto.

Os modelos de regressão tiveram como variável dependente a permeabilidade a como variáveis independentes 
os perfis GR, DT e DRDN. DRDN é uma relação linear entre os perfis RHOB e NPHI, a qual quantifica a separação entre eles numa escala adequada (Equação 2).

$$
D R D N=\frac{R H O B-2}{0.05}-\frac{0.45-N P H I}{0.03} .
$$

Dos 493 valores foram eliminados aqueles com permeabilidade inferior a $0,1 \mathrm{mD}$ ou por estarem fora do intervalo do estudo. Os intervalos utilizados para a construção das estimativas compreendem as profundidades entre $1796 \mathrm{e}$ 1850 m (poço X3) e 1758 e 1875 m (poço X10). Somente a terceira Ksint é mostrada na Equação 2 a qual contem 266 observações do poço $\mathrm{X} 10$ e teve o mais alto $\mathrm{R}^{2}=$ 0.472 .

$$
\begin{aligned}
& K \sin t=10^{\wedge}(-0.71829066-0.17741662 G R \\
& -0.37186828 D R D N+0.05796762 D T)
\end{aligned}
$$

Para que as petrofácies fossem determinadas, foram calculados os raios de garganta de poro (Kolodzie, 1980), para a saturação acumulada de $35 \%$ de mercúrio (R35), a fim de representar as curvas capazes de distinguir as energias dos ambientes deposicionais (Winland, 1972; Pittman, 1992). Foram escolhidas três unidades que indicam a qualidade permoporosa da rocha em termos do conceito de petrofácies: boa (grainstones e packstones porosos), regular (grainstones e packstones oncolíticos, peloidais e microoncolíticos/microolítocos) e ruim (packstones muito finos, mudstones, wackstones e cimentação carbonática).

\section{Resultados}

A eliminação dos valores inferiores a $0,1 \mathrm{mD}$ de permeabilidade de plug tenta evitar algum viés que possíveis erros de medição ou de carregamento no banco de dados venham a causar. O gráfico de porosidade versus permeabilidade de plug, contendo os 512 pares de valores (poços X03 e X10), realça a distribuição anômala desses valores (Figura 1). O mesmo acontece os valores de $0.2 \mathrm{mD}, 0.3 \mathrm{mD}$ e $0.4 \mathrm{mD}$, os quais não foram retirados. Deve ser destacado que Ksint fornece cálculos mais pessimistas para os altos valores e mais otimistas para os baixos, devido à redução da variância quando observada pelo $\mathrm{R}^{2}$. Como se deseja uma correta avaliação das rochas que são reservatórios, esta abordagem pode ser considerada como pessimista do ponto de vista da análise de incerteza.

Nas Figuras 2 e 3 são mostrados os perfis dos poços X3 e X10, contendo o conjunto básico de perfis, as litofácies descritas, a eletrofácies discriminante, a porosidade total de perfil, Ksint e os dados da petrofísica básica. Os códigos de cores que aprecem nessas figuras aparecem na Figura 4. Nestas figuras se observa como a dispersão dos pontos em relação à curva média que representa a permeabilidade de plug ficou maior para os dados do poço X3, que obteve o menor valor de $R^{2}$ na regressão. Esse fato levaria à necessidade de uma investigação petrográfica mais detalhada. A escolha de um modelo matemático mais ajustado para o cálculo de Ksint é importante para a extrapolação das petrofácies aos poços que não possuem testemunhos, fornecendo maior confiabilidade na sua predição (track 11 das Figuras 2 e 3). A porosidade de perfil é relativamente mais confiável e costuma ter boa correlação com a porosidade de plug.

Nestes perfis, as curvas de GR e DRDN foram discretizadas na tentativa de representar eletrofácies através de intervalos de valores, conforme mostrado nas legendas da Figura 4. Neste momento, é possível realizar uma correlação visual entre as fácies (litofácies e eletrofácies discriminantes) com os respectivos comportamentos em perfil e que aparecem resumidos na Tabela 2. A descrição contida nesta tabela é uma tentativa aproximada de prever a fácies de uma maneira bastante simples, levando pouco em consideração o fluido contido e a diagênese associada.

A porção que se encontra entre 1.2 e 5.5 micras, na faixa de permeabilidade aproximada entre 10 e $100 \mathrm{mD}$, pode ser considerada como as petrofácies regular (packstones mais finos e grainstones oncolíticos a microoncolíticos) enquanto que abaixo de 1.2 micras estão as petrofácies ruim (fácies cimentada, mudstones e talvez alguns wackestones) com permeabilidades inferiores a $10 \mathrm{mD}$. Pela análise da Figura 5 foi possível distinguir, através dos cortes utilizados e com a limitação do pequeno conjunto de dados, três regiões com diferentes características permoporosas: petrofacies boa, regular e ruim. Essa figura mostra os cortes e as três petrofacies mencionadas. Vale ressaltar que a boa correlação entre o raio de garganta de poro e a permeabilidade pode explicar a preferência do sistema poroso ao fluxo de fluidos quando saturado em $35 \%$.

\section{Conclusões}

Ao se avaliar o comportamento dos perfis, nota-se que as fácies que possuem valores extremos como $G R$ alto $x$ GR Baixo, RHOB alto x RHOB baixo, por exemplo, são bem separadas por todas as técnicas. Porém, quando o perfil apresenta comportamento intermediário ou aquelas que um determinado perfil tem um peso maior, a classificação das fácies se torna mais distribuída entre os diversos grupos. No grupo da fácies grainstones aparecem poucos mudstones e vice-versa, porém naqueles intermediários grain/packstone e pack/grainstone ocorre um espalhamento dos pontos. Na verdade, ocorrem nuances texturais entre grainstones e packstones, e, packstones e mudstones. Essas características afetam significativamente a permo porosidade da rocha. Para a construção de um modelo de fluxo, neste caso, o emprego de 3 petrofácies e 1 fácies diagenética seria o mais apropriado. Considerando que as litofácies refletem essencialmente o controle deposicional da rocha sem incluir na sua classificação textural e os fenômenos diagenéticos, podemos considerar que, para as regiões dos testemunhos não afetadas pela diagênese, existe uma boa correlação entre as litofácies grainstone com a petrofácies 
boa, packstones com a petrofácies regular e litofácies mudstones/wackstone com a petrofácies ruim.

\section{Agradecimentos}

Agradecemos à UENF/LENEP pela infraestrutura computacional, CNPq pela bolsa de pesquisa, LR Senergy pela licença acadêmica de software IP e a Petrobras/ANP pelo conjunto de dados reais e recursos para pesquisa.

\section{Referências}

Doveton J. 1994. Geologic log analysis using computer methods. Am. Assoc. Petroleum Geologists, Computer Methods in Geology, n. 2, 169 p.

Kolodzie, S. 1980. Analysis of pore throat size and use of Waxman-Smits equation to determine OOIP in Spindle Field, SPE Annual Technical Conference and Exhibition, Dallas, 21-24 sept. Paper 9382. ciation of Petroleum Geologist. Bulletin 76, nำ2, Tulsa, p. 191-198.

Ellis, D. \& Singer, J. 2007. Well logging for earth scientists: Springer.

Lucia, J. 2007. Carbonate reservoir characterization, an integrated approach. Springer-Verlag Berlin Heidelberg, $341 \mathrm{p}$.

Rider, M. The geological interpretation of well logs. (Second Edition) Sutherland, Scotland: Rider-French Consulting Ltd, 2000. 280 p.

Schlumberger. 1987. Log interpretation: Principles / Applications. Schlumberger Ltd., New York, 198 p.

Serra, O. \& Abbott, H. 1982. The contribution of logging data to sedimentology and stratigraphy. Society of Petroleum Engineers Journal, 5, 6, 29.

Serra, O. 1986. Sedimentary environments from wireline logs. Schlumberger, New York, 211p.

Windland, H. 1972, Oil accumulation in response to pore size changes, Weyburn Field, Saskatchewan: Amoco Production Research Report, No. F72-G-25.

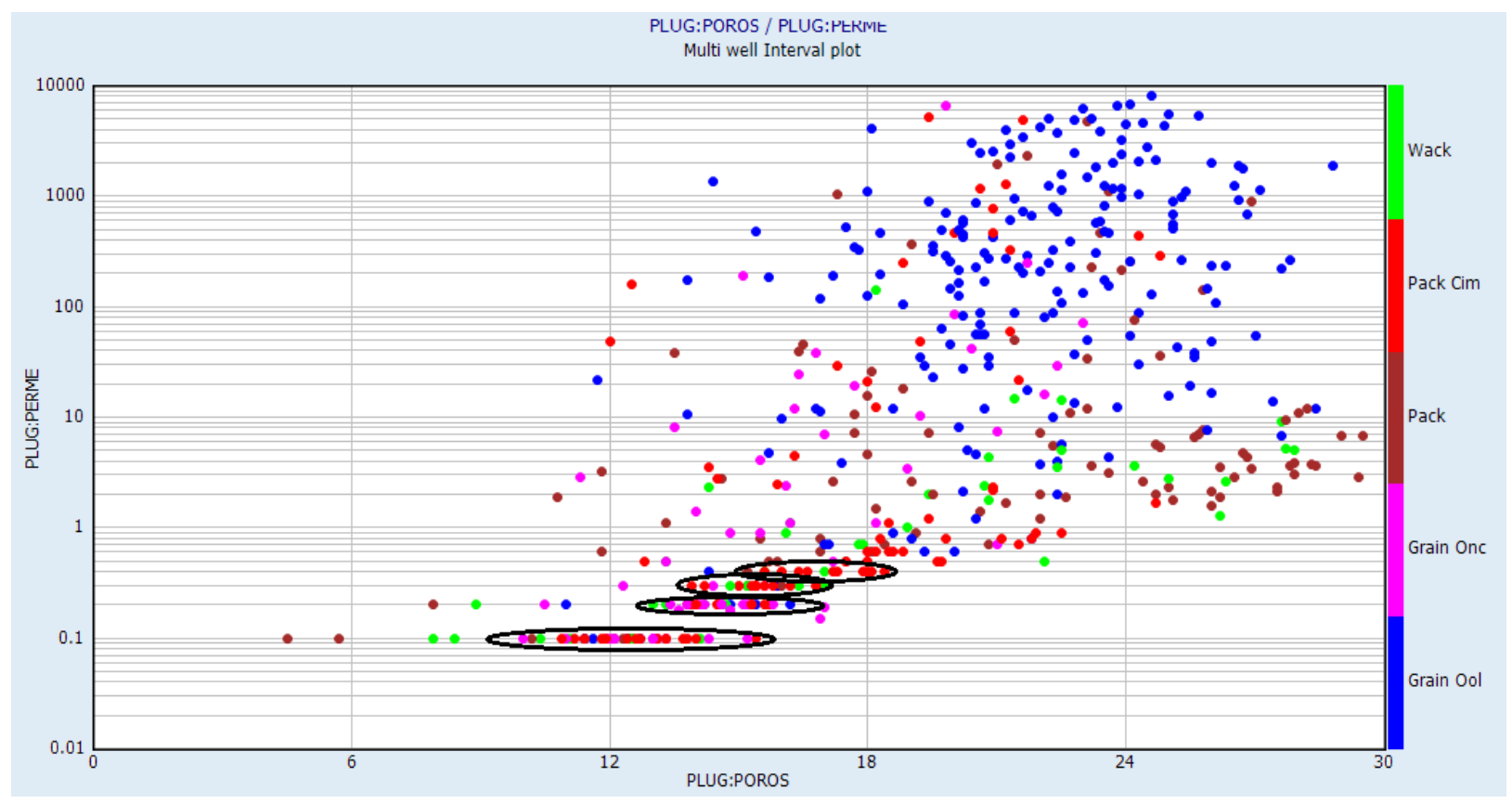

Figura 1. Porosidade versus permeabilidade, ambos obtidos por medidas de laboratório, mostrando a distribuição anômala, destacadas em preto, dos valores de $0.1 \mathrm{mD}, 0.2 \mathrm{mD}, 0.3 \mathrm{mD}$ e $0.4 \mathrm{mD}$. As cores dos marcadores representam as eletrofácies discriminantes.

Pittman, E. 1992. Relationship of porosity and permeability to various parameters derived from mercury injection capillary pressure curves for sandstone, American Asso- 


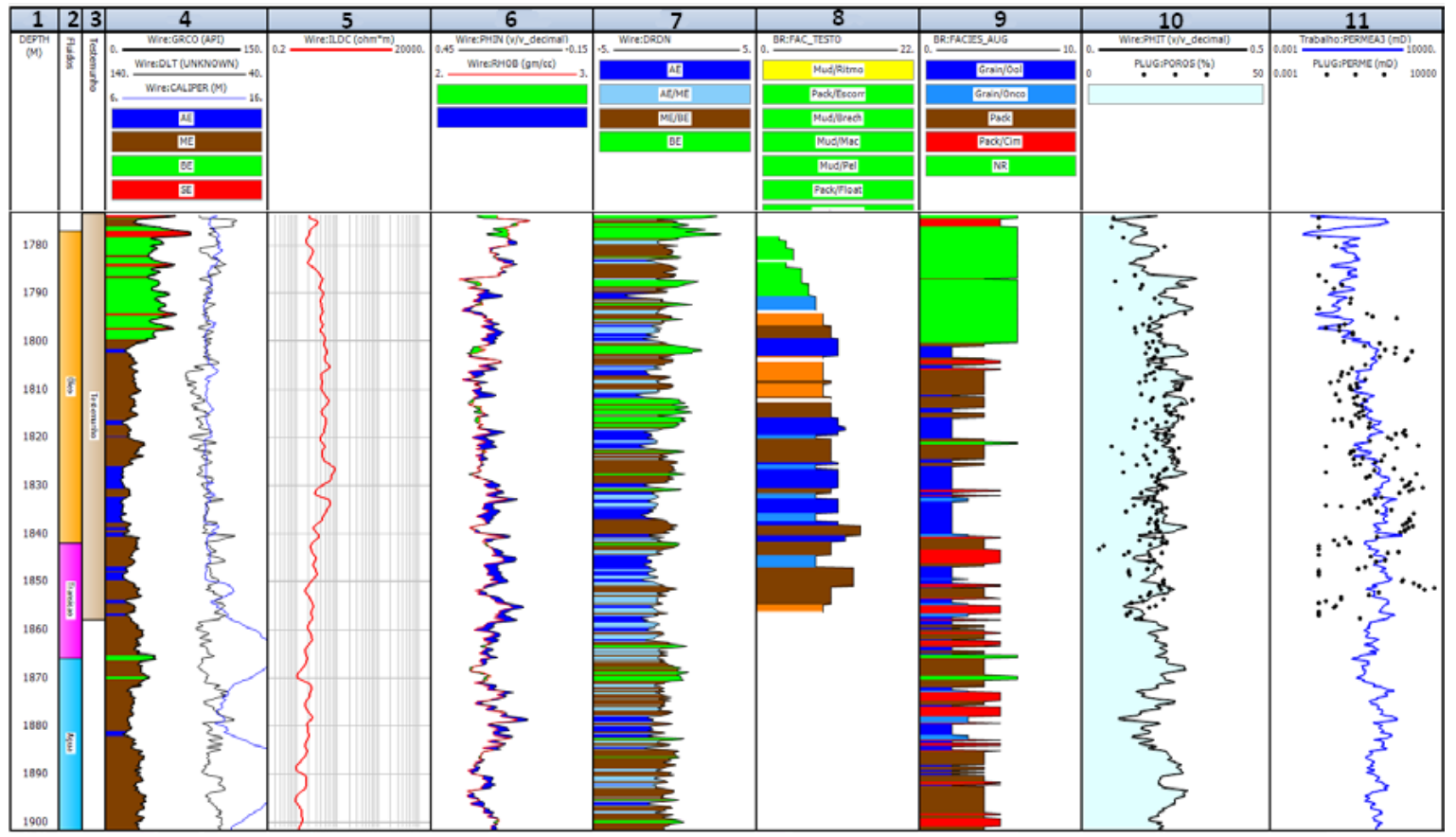

Figura 2. Resumo dos dados de perfis geofísicos do poço X3 entre 1780 e $1900 \mathrm{~m}$. Descrição das colunas: 1 - Profundidade $(\mathrm{m}), 2$ - Fluidos do reservatório, 3 - Profundidade com dados de testemunho entre $1770 \mathrm{~m}$ e 1857m, 4 - Resistividade profunda (ohmm), 5 - Densidade $\left(\mathrm{g} / \mathrm{cm}^{3}\right)$ Neutrônico (v/v), 6 - Raios Gama (API), Caliper $(\mathrm{m})$ e Sônico ( $\mu^{*} \mathrm{~s} /$ pé), 7 - DRDN (adimensional), 8 - Litofácies descritas (adimensional), 9 - Eletrofácies discriminante (adimensional), 10 - Porosidade total (v/v) e porosidade de plug (\%) e 10 - Permeabilidade sintética $(\mathrm{mD})$ e permeabilidade de plug $(\mathrm{mD})$.

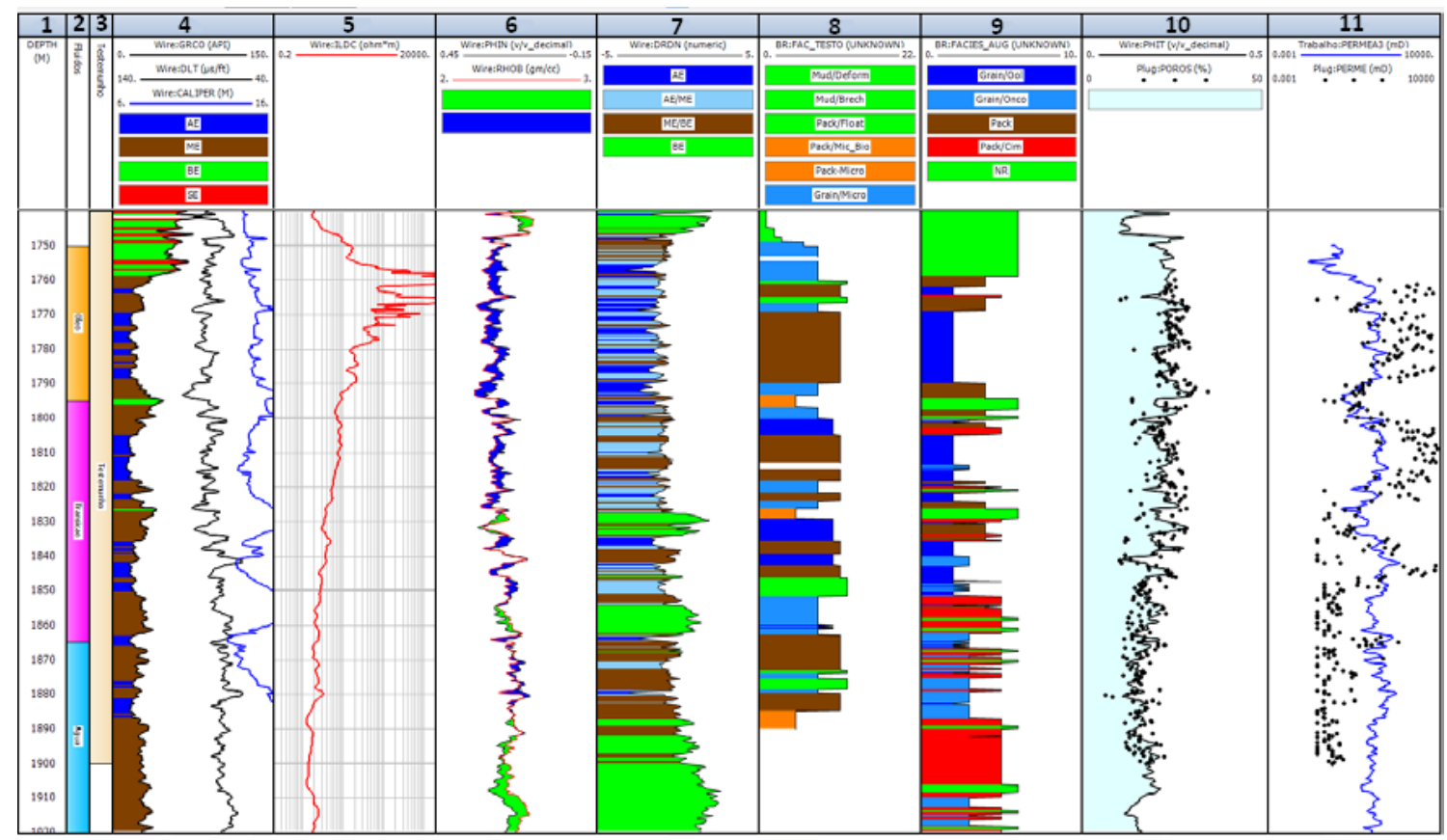

Figura 3. Resumo dos dados de perfis geofísicos do poço X10 entre 1740 e $1910 \mathrm{~m}$. Descrição das colunas: 1 - Profundidade $(\mathrm{m}), 2$ - Fluidos do reservatório, 3 - Profundidade com dados de testemunho entre $1770 \mathrm{~m}$ e $1857 \mathrm{~m}, 4$ - Resistividade profunda(ohm $\left.{ }^{*}\right), 5$ - Densidade $\left(\mathrm{g} / \mathrm{cm}^{3}\right)$ Neutrão (v/v), 6 Raios Gama (API), Caliper $(\mathrm{m})$ e Sônico $\left(\mu^{*} \mathrm{~s} /\right.$ pé), 7 - DRDN (adimensional), 8 - Litofácies descritas (adimensional), 9 - Eletrofácies discriminante (adimensional), 10 - Porosidade total $(\mathrm{v} / \mathrm{v})$ e porosidade de plug $(\%)$ e 10 - Permeabilidade sintética $(\mathrm{mD})$ e permeabilidade de plug $(\mathrm{mD})$. 
(a) Discretização GR

\begin{tabular}{|l|l|l|l|}
\hline \multicolumn{1}{|c|}{ Low Value } & \multicolumn{1}{|c|}{ High Value } & Shading Type & \multicolumn{1}{c|}{ Description } \\
0 & 20 & & AE \\
\hline 20 & 40 & \\
\hline 40 & 60 & ME \\
\hline 60 & 80 & & BE \\
\hline
\end{tabular}

(b) Discretização DRDN

\begin{tabular}{|l|l|l|l|}
\hline \multicolumn{1}{|c|}{ Low Value } & \multicolumn{1}{c|}{ High Value } & \multicolumn{1}{c|}{ Shading Type } & \multicolumn{1}{c|}{ Description } \\
& -1.3 & & AE \\
\hline-1.301 & -0.8 & & AE/ME \\
\hline-0.8001 & 0.2 & & ME/BE \\
\hline 0.2001 & 5 & & BE \\
\hline
\end{tabular}

(c) Eletrofácies Discriminante

\begin{tabular}{|l|l|l|l|}
\hline \multicolumn{1}{|c|}{ Low Value } & \multicolumn{1}{c|}{ High Value } & \multicolumn{1}{c|}{ Shading Type } & \multicolumn{1}{c|}{$\begin{array}{l}\text { Description } \\
1.5\end{array}$} \\
& 2.5 & & Grain/Ool \\
\hline 2.5 & 3.5 & & Grain/Onco \\
\hline 3.5 & 4.5 & & Pack \\
\hline 4.5 & 5.5 & & Pack/Cim \\
\hline 5.5 & 6.5 & & NR \\
\hline
\end{tabular}

(d) Poço X10 - Litofácies

\begin{tabular}{|c|c|c|c|c|c|c|c|}
\hline Low Value & High Value & Shading Type & Description & Low Value & High Value & Shading Type & Description \\
\hline 0.5 & 1.5 & & Mud/Deform & 0.5 & 1.5 & & Mud/Ritmo \\
\hline 1.5 & 2.5 & & Mud/Brech & 1.5 & 2.5 & & Pack/Escorr \\
\hline 2.5 & 3.5 & & Pack/Float & 2.5 & 3.5 & & Mud/Brech \\
\hline 3.5 & 4.5 & & Pack/Mic_Bio & 3.5 & 4.5 & & Mud/Mac \\
\hline 4.5 & 5.5 & & Pack-Micro & 4.5 & 5.5 & & Mud/Pel \\
\hline 5.5 & 6.5 & & Grain/Micro & 5.5 & 6.5 & & Pack/Float \\
\hline 6.5 & 7.5 & & |Grain_Ool & 6.5 & 7.5 & & Pack OcBio \\
\hline 7.5 & 8.5 & & Grain/Ool & 7.5 & 8.5 & & | Pack Grain/Micro \\
\hline 8.5 & 9.5 & & Grain/Mic_Ool & 8.5 & 9.5 & & Pack/Pel \\
\hline 9.5 & 10.5 & & | Grain/Ool & 9.5 & 10.5 & & $\|$ Grain Pack/Oc \\
\hline 10.5 & 11.5 & & Pack/Oc & 10.5 & 11.5 & & | Grain/Ool \\
\hline 11.5 & 12.5 & & Mud/Pel & 11.5 & 12.5 & & ||Grain/OolMicro \\
\hline 12.5 & 13.5 & & MRG & 12.5 & 13.5 & & U Grain/Oc \\
\hline 13.5 & 14.5 & & | Grain/Ool & 13.5 & 14.5 & & |lPack/Oc \\
\hline
\end{tabular}

Figura 4. Legendas em cores: a - Discretização do GR para os poços X3 e X10 (AE = Alta Energia, ME = Moderada Energia, $\mathrm{BE}=$ Baixa Energia e SE = sem energia), $\mathrm{b}$ - Discretização do DRDN para os poços X3 e X10 (AE = Alta Energia, $\mathrm{AE} / \mathrm{ME}$ = Alta a Moderada Energia, $\mathrm{ME} / \mathrm{BE}=$ Moderada a Baixa Energia e $S E=$ sem energia), $c$ - Eletrofácies discriminante para os poços X3 e X10, d - Litofácies descritas no poço X10 e e - Litofácies descritas no poço X3. As curvas discretizadas GR e DRDN tentam representar a energia deposicional (AE - alta energia - Grainstones, ME - moderada energia - packstones e BE - Baixa energia - mudstones).

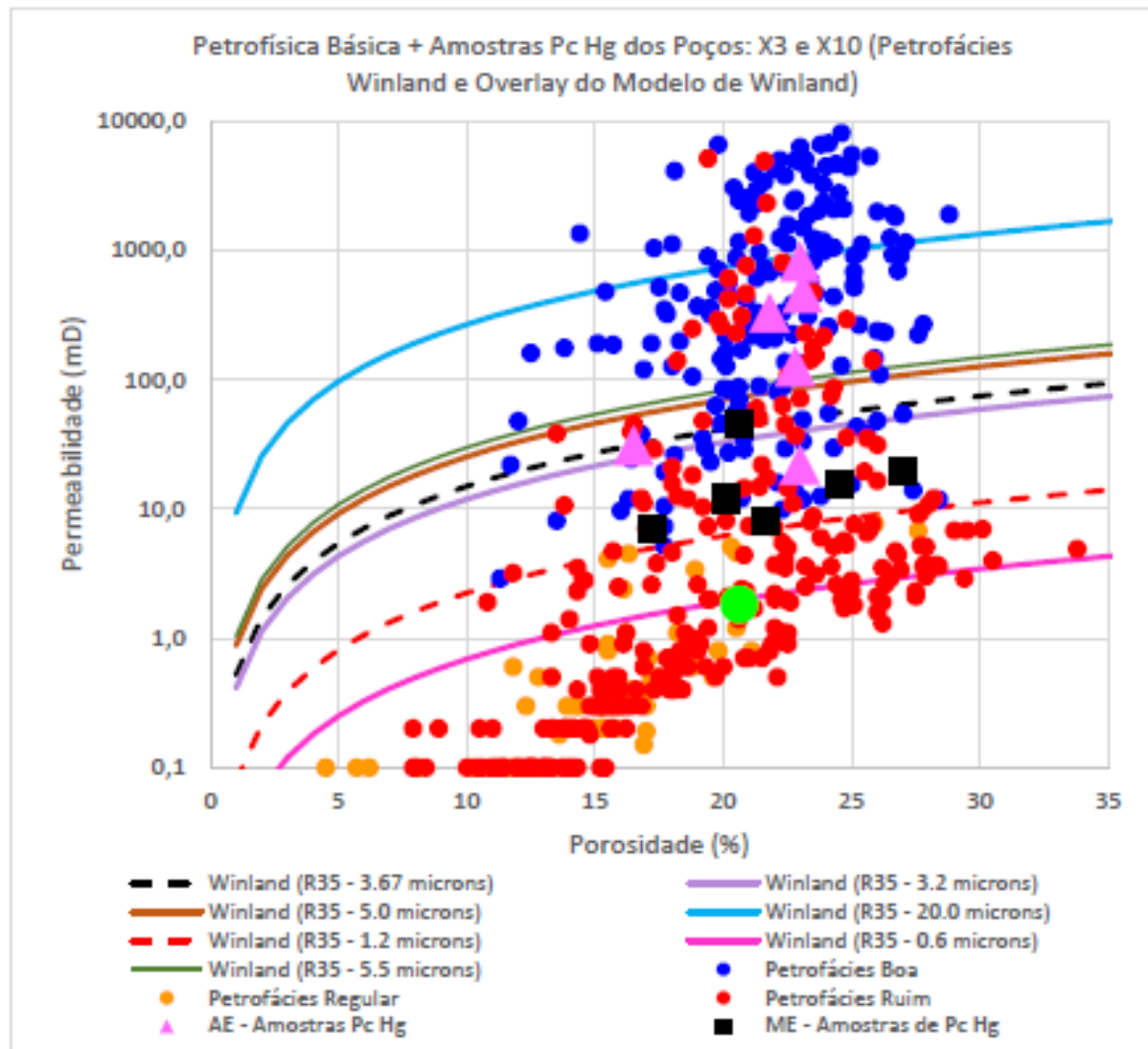

Figura 5. Porosidade (\%) versus permeabilidade $(\mathrm{mD})$ medidas em laboratório mostrando três grupos de Petrofácies, ensaios de pressão capilar por injeção de mercúrio e as curvas de Winland para 0 poço X3 e X10. Foram utilizados os valores de corte de raios de garganta de 1.2 microns (linha vermeIha tracejada) e 3.67 microns (linha preta tracejada). 
Tabela 1. Perfis utilizados para a interpretação de eletrofácies e sua descrição do comportamento médio esperado para os perfis GR, RHOB e DT.

\begin{tabular}{|c|c|c|c|}
\hline PROVÁVEL FÁCIES & GR & RHOB & DT \\
\hline Grainstone & baixo & baixo a médio & médio a alto \\
\hline Packstone & baixo & baixo a médio & médio a alto \\
\hline Cimento & baixo & alto & baixo \\
\hline Mudstone & alto & baixo a médio & médio a alto \\
\hline Wackstone & alto & alto & baixo \\
\hline
\end{tabular}

